\title{
Measurements of X-ray radiation of the Sun on the heliogeophysical measuring equipment of spacecraft of the Russian Hydrometheorological Service
}

\author{
Anatoly Nusinov ${ }^{1}$, Vladimir Minligareev ${ }^{1}$, Andrey Repin ${ }^{1}$, and Igor Shirokov ${ }^{2, *}$ \\ ${ }^{1}$ E. K. Fedorov Institute of Applied Geophysics, Moscow, Russia \\ ${ }^{2}$ Sevastopol State University, Sevastopol, Russia
}

\begin{abstract}
The characteristics of the equipment for monitoring X-ray solar radiation onboard geostationary satellites. A reference base for X-ray flux meters of the DIR-E was developed and experimental studies of the characteristics on the technological sample of the device were carried out. The calibration procedure of DIR-E for use in measurements on perspective space vehicles was also developed.
\end{abstract}

\section{Introduction}

Ones of the most important objects of space weather monitoring are the solar flares, which have a significant effect on the upper atmosphere and ionosphere. A solar flare is accompanied by a relatively short-term strong increase in the soft X-ray flux (SX) in the range 0.01-10 nm and a much weaker increase in flux in upper ultraviolet radiation (UUV, 10-105 nm). Changes in the ionosphere (in its lighted part) accompanying such phenomena are usually called sudden ionosphere disturbances.

The solar flare may be accompanied by an increase in the flux of high-energy protons, or so-called solar cosmic rays (SCR), which are also the source of anomalous ionization of the D-region of the ionosphere (the so-called polar cap absorption, PCA). The effects of ionosphere disturbances and PCA significantly change the conditions of propagation of radio waves and these effects have an important influence on space weather. Mentioned factors determine the importance of observing fluxes of ultraviolet and X-rays from the entire Sun as a star, as well as precipitations of high-energy particles. In addition, the importance of measuring soft X-rays is due to the fact that it is an extremely sensitive indicator of solar processes leading to geomagnetic disturbances. So, one of the important applications of simultaneous measurements of X-rays and UUV is the estimation the proton fluxes of solar flares.

At the same level of the SX flux, proton events are accompanied by UUV streams several times larger than in the absence of protons. In [1], relations are given that make it possible to separate proton and non-proton events, based on data on the UUV fluxes and SX fluxes. This allows us to estimate the fluxes of protons by the measurements of UUV radiation.

*Corresponding author: shirokov@ieee.org 
It has to be understood the delay between appearance of X-ray flux and particles bombing of Earth atmosphere lies in time interval from several hours till several days. So, in-time determination of X-ray flux plays an important role in overall protection of the electronic equipment of humanity use.

Thus, by using simultaneous measurements of SX and ultraviolet radiation, it becomes possible to recognize radiation-dangerous events on the Sun and to estimate the flux of protons almost at the moment radiation arrives from the solar flare to the Earth.

\section{State-of-art fluxes measurements}

The processing of GPS / GLONASS signals shows that during flares up to $30 \%$ of the increment of the total electron content (TEC) occurs at heights $h>300 \mathrm{~km}$. In other words an increase in the electron concentration $N$ occurs at all altitudes of the ionosphere, and the relative contribution of the external ionosphere is significant.

For decades, measurements on spacecrafts (SC) have led to the selection of the most convenient measurement band for monitoring. Systematic X-ray measurements have been carried out on US spacecrafts for almost 50 years. At present, observations are carried out on geostationary SC of the "GOES" series in the wavelength ranges $0.05-0.4 \mathrm{~nm}$ and 0.1 $0.8 \mathrm{~nm}$. The classification of X-ray flashes and background radiation level is carried out by the magnitude of the energy flux $I_{8}$ (range $0.1-0.8 \mathrm{~nm}$ ) and the energy flux $I_{4}$ (range 0.05$0.4 \mathrm{~nm}$ ), or as it is so-called X-ray class.

Only class $\mathrm{C}$ flares can produce noticeable ionosphere effects (energy flux in 1$9 \mathrm{uW} / \mathrm{m}^{2}$ and more). Currently, the accuracy of measurements of X-ray fluxes is becoming more and more high, and the measurement error in the region of energies $\sim 3 \mathrm{keV}$ does not exceed $\sim 20 \%$.

Due to the fact that the radiations forming the ionosphere and the upper atmosphere are completely absorbed already at altitudes in $\sim 90 \mathrm{~km}$, their measurements are fundamentally possible only on a SC.

On the Russian geostationary SC of the type "Electro-L" (Fig. 1) the DIR-E solar X-ray flux meters are installed for measuring the X-ray flux density of the Sun in the energy range from 3 to $25 \mathrm{keV}(0.05-0.4 \mathrm{~nm})$. These characteristics do not allow to directly carrying out the classification of flares in a generally accepted way all over the world. Also it is impossible to compare measurements with the results of observations of the GOES satellite.

The equipment for measuring of UUV (VUSS-E) and X-ray (DIR-E) radiation on geostationary SC "Electro-L" are installed on the solar panel bracket. The UUV meter was designed for measuring the energy of flux density of the UV radiation of the Sun near the $H L \alpha$ line $(121.6 \mathrm{~nm})$ in the range $1-30 \mathrm{~mW} / \mathrm{m}^{2}$, and one operates in conjunction with DIR-E.

The design of the SC provides the permanent tracking by the solar panels to the Sun with an acceptable deviation of the normal vector to the plane of the solar battery from the direction to the Sun in a range $7-8^{\circ}$. Since the directivity pattern of the instrument is not isotropic and one strongly depends on the angle between the direction to the Sun and the axis of the instrument, a correction of the instrument readings should be provided.

\section{Problem to be solved}

As the X-ray sensor the DIR-E uses a Geiger counter. The difference from the measurements on the geostationary US spacecraft is that the GOES measurements are carried out using two ionization chambers [2] with different bands $(0.05-0.4 \mathrm{~nm}$ and $0.1-0.8 \mathrm{~nm})$. Measurements in two X-ray bands allow determining the temperature and the measuring of emission in accordance with the method proposed in [3]. One of the latest options for improving the method, which is based on new spectrum calculations, was proposed in [4]. 

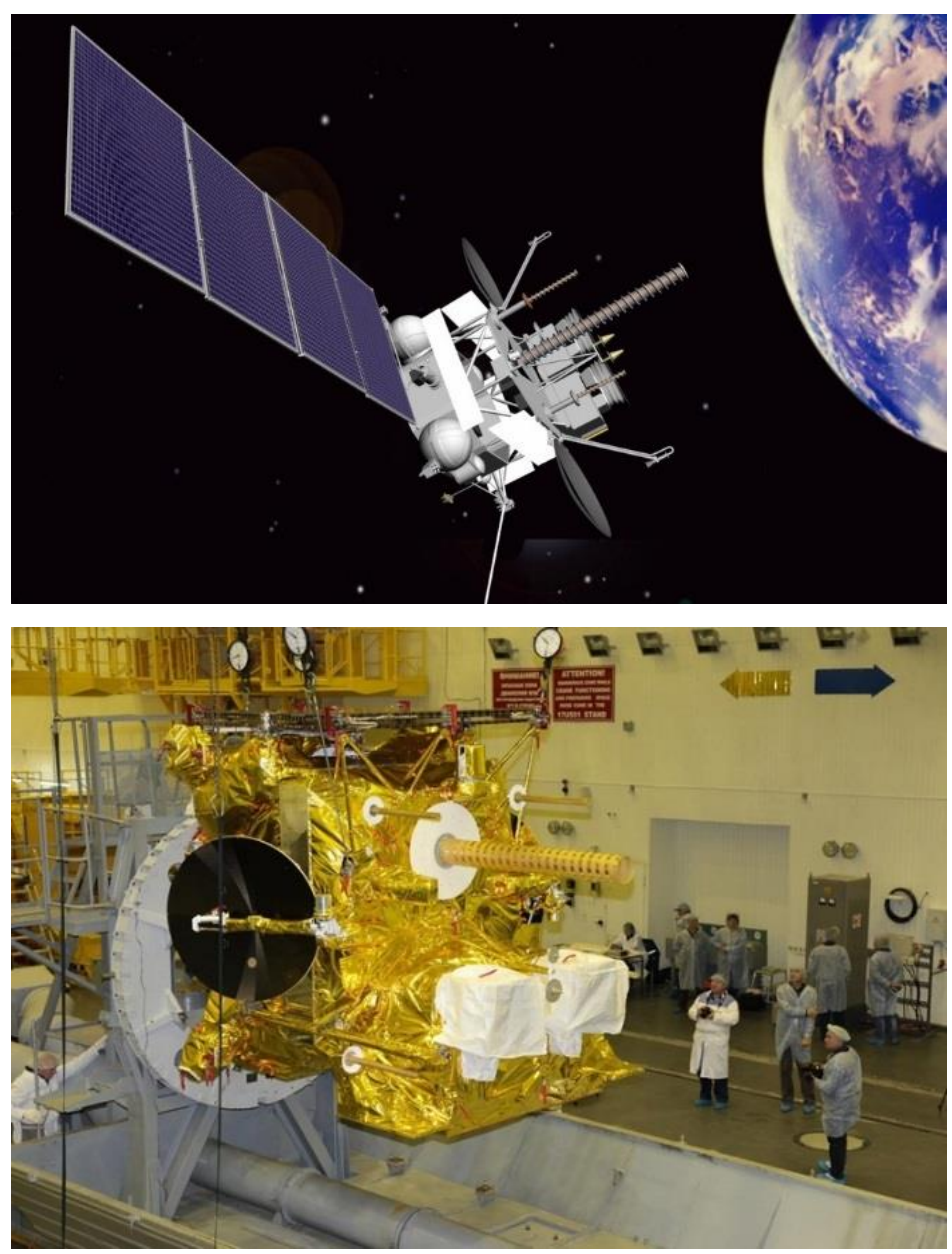

Fig. 1. Geostationary SC "Electro-L".

Since measurements of SX on the SC "Electro-L" are carried out, in contrast to GOES, using a Geiger counter, it is necessary to develop a method for determining the radiation energy flux based on the counting rate.

At present, the theoretical spectra from the modern CHIANTI database $[5,6]$ are used to work with GOES data. It was shown in [7] the spectrum calculated on the basis of the full accounting of all radiation processes (free-free transitions, free-bound transitions, dielectron recombination and line emission) can be calculated with an accuracy of about $15 \%$ based on a simple radiating losses function,

$$
L(E, T)=2.59 \cdot 10^{-20} \frac{1}{\sqrt{T}} \exp \left(-\frac{E}{k T}\right),
$$

where $E$ is the quantum energy, $k$ is the Boltzmann constant, $T$ is the plasma temperature.

By integrating (1) over the energy from $E$ to $\infty$ one can obtain flows $I_{8}$ and $I_{4}$ for each of the intervals:

$$
I_{8}=A \sqrt{T} \exp \left(-\frac{E_{8}}{k T}\right), \quad I_{4}=A \sqrt{T} \exp \left(-\frac{E_{4}}{k T}\right),
$$


where the coefficient $A$ includes the measure of emission and the attenuation of radiation with distance; $E_{8}$ and $E_{4}$ is the energy of quanta at a wavelength corresponding to the upper boundary of each of the intervals.

From (2), the temperature $T$ can be determined if the fluxes $I_{8}$ and $I_{4}$ are known:

$$
k T=\frac{E_{4}-E_{8}}{\ln \left(I_{8} / I_{4}\right)} .
$$

The relationship between these fluxes follows a power law and it is obtained according to data from various SC GOES [8] for various flares:

$$
I_{8}=0.2009 I_{4}^{0.72}
$$

where flux values are expressed in $\mathrm{W} / \mathrm{m}^{2}$. This ratio is obtained for flares of all classes (from A to X) and can be refined by splitting flares into separate classes from A to X. Then, the counting rate of the detector $V$ can be written as

$$
V=Z \int_{0}^{4} S(\lambda) N_{\lambda} d \lambda,
$$

where $Z$ is the area of the counter window; $S(\lambda)$ is its sensitivity; $N_{\lambda}$ is the spectral function for a given $T$.

The integral (5) includes the sensitivity function $S(\lambda)$. The values of this function at various wavelengths $\lambda$ were determined experimentally during calibration of devices.

\section{Calibration of the device DIR-E}

A necessary condition for the quality of heliogeophysical monitoring is the achieving the required accuracy, reliability and comparability of measurement results. A significant improvement in the quality of heliogeophysical observations can be achieved only if the metrological support system (MSS) of the target equipment of SC is implemented.

At the Institute of Applied Geophysics named after academician E. K. Fedorov the work for creation a distributed reference base for calibrating target equipment for heliogeophysical purposes, including the DIR-E meter was carried out $[9,10]$. As a result of the work on the creation of a reference base, a workplace for the DIR-E solar X-ray flux meter was created. At the workplace the DIR-E calibration based on photon flux density was performed by direct measurements in X-ray fields of reference radionuclide sources ${ }^{55} \mathrm{Fe}$ and ${ }^{109} \mathrm{Cd}$. The calibration was also performed by replacing the detector of the reference radiometric equipment certified for photon flux density in the low-energy X-ray field of a reference standard of the state dosimeter standard (SDS). Calibration with a generating X-ray source was carried out at average radiation modes from 5 to $21 \mathrm{keV}$ in the photon flux density range from $10^{2}$ to $10^{5} \mathrm{~cm}^{-2} \mathrm{~s}^{-1}$.

Testing calibration techniques on the SDS was carried out in the X-ray range $5-23 \mathrm{keV}$. The following standards were applied:

— working standard of 1 category XFe 5.24 based on ${ }^{55} \mathrm{Fe}$ radionuclide;

— working standard of 1 category $\mathrm{XCd} 9.06$ based on ${ }^{109} \mathrm{Cd}$ radionuclide;

- X-ray equipment UED 5-50M.

In the process of calibration work, the sensitivity of the instrument was determined. The sensitivity was the ratio of the change in the pulse count rate of the measuring channel to the change in beta-particle or photon flux density. The calibration was carried out at one or 
several values of the flux density of particles (photons) in a certain range of radiation energies and for several angles of setting the device relative to the geometric axis of the flux of particles (photons).

The results of the calibration works of the DIR-E instrument confirmed the correctness of the choice of methods for evaluating the metrological characteristics of the instruments and the nomenclature of the reference measuring instruments used at the calibration. The results of calibration of two devices on the set of standard means are shown in Fig.2. The dotted line denotes the approximation by a spline function (sensitivity function $S(\lambda)$ ).

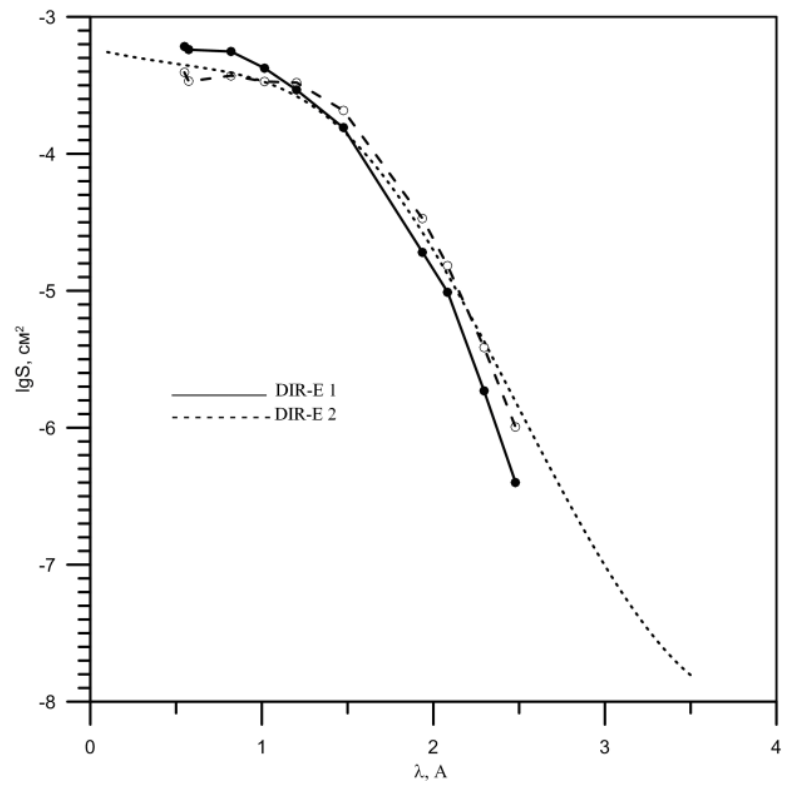

Fig. 2. The results of the calibration devices DIR-E.

The results obtained when calibrating DIR-E:

— sensitivity of the device was $1.0 \cdot 10^{-6}-3.9 \cdot 10^{-4} \mathrm{~cm}^{2}$;

— extended uncertainty of sensitivity measurement result $(\mathrm{k}=2)$ was $12 \%$.

\section{The readings of the device DIR-E with a change in the solar X-ray flux}

The knowledge of the sensitivity function $S(\lambda)$ makes it possible to calculate the response of the device to the X-ray flux using equation (5).

The detector response calculations were carried out for different flare points: from B1 $\left(I_{8}=10^{-7} \mathrm{~W} / \mathrm{m}^{2}\right)$ to $\mathrm{X} 9\left(I_{8}=910^{-4} \mathrm{~W} / \mathrm{m}^{2}\right)$. The area of the $Z$ detector was assumed to be $0.05 \mathrm{~cm}^{2}$, which corresponds to the size of the diaphragm in front of the counter window. The calculation results are shown in Fig. 3. It can be seen the dependence of the flare point on the meter readings can be well described by the power dependence; it corresponds to the dotted line in Fig. 2.

The ratio

$$
I_{8}=4.12 \cdot 10^{-7} \cdot V^{0.73}
$$

corresponds to the approximating dotted line in Fig. 3 and can be used for estimating the flare score from the readings of an X-ray detector based on a Geiger counter. 


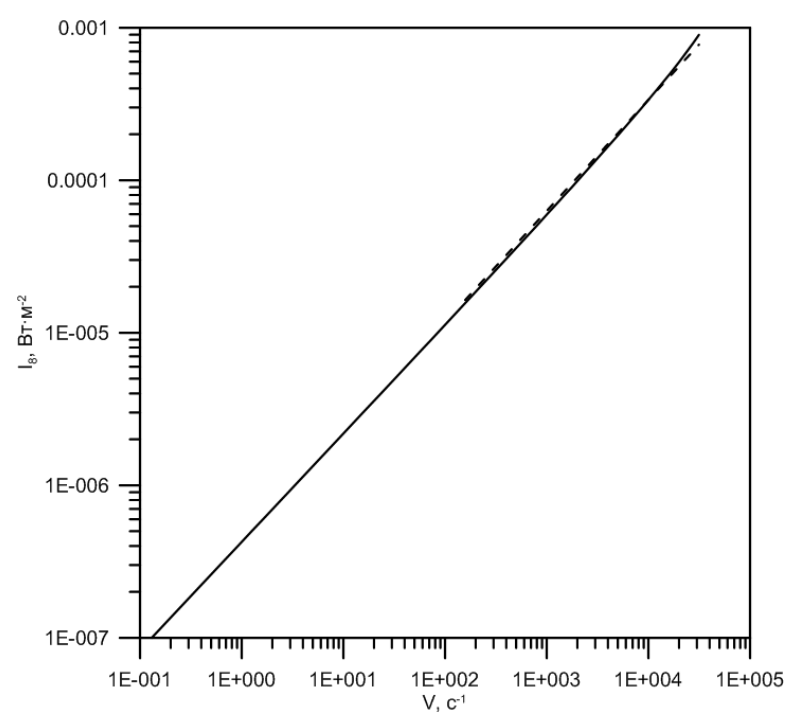

Fig. 3. Dependence of the flare point on the counting rate of the $\mathrm{X}$-ray detector.

It is necessary to take into account that all the above estimates relate only to the situation when the detector axis is directed to the Sun. In terms of measurements, when the angle between the axis of the detector and the direction to the Sun is continuously changing, it is necessary to introduce an amendment to the dependence of the sensitivity from the angle.

\section{Conclusions}

A method for processing measurements for solar X-ray flux meters DIR-E of geostationary SC "Electro-L" and the subsequent reduction of these measurements to the standard internationally accepted form as the energy flow in the range of $0.1-0.8 \mathrm{~nm}$ has been developed.

The reference base for DIR-E has been developed and experimental characteristics have been studied on a technological instrument sample. A DIR-E calibration method has been developed.

\section{References}

1. L. A. Antonova, A. A. Nusinov, The Use of measurements of upper ultraviolet radiation from the Sun to diagnose the radiation hazard of solar flares, News of Academy of Science RF, ser. Physical, Vol.59, No. 8, P.76-80 (1995)

2. R. U. Kreplin, K. R. Dir, D. M. Khoran, J. F. Mikins, The spectrum of the Sun in a band shorter than 10 A, in the book The Sun Energy Flow and Its Changes. Ed. O.R. White, Moscow, Mir, P. 313-339 (1980)

3. H. A. Garcia, Temperature and GOES soft X-ray measurements, Solar Physics, Vol. 154, No. 2, P. 275-308 (1994)

4. S. M. White, R. J. Thomas, R. A. Schwartz, Updated X-ray measurements, Solar Physics, Vol. 227, No. 2, P. 231-248 (2005)

5. E. Landi, U. Feldman, K. P. Dere, CHIANTI: an Atomic Database for Emission Lines. $V$. Comparison with an Isothermal Spectrum Observed with SUMER, The Astrophysical Journal Supplement Series, Vol. 139, Issue 1., P. 281-296 (2002) 
6. E. Landi, M. Landini, K. P. Dere, P. R. Young, H. E. Mason, CHIANTI: an atomic database for emission lines. Iii. Continuum radiation and the ion database, Astronomy and Astrophysics Supplement, Vol.135, P.339-346 (1999)

7. B. V. Somov, About the contribution of X-ray radiation from a solar flare to the heating of its low-temperature (optical) region, in the book: Cosmic rays in the stratosphere and in near-Earth space, Proc. Physical Institute of the USSR Academy of Sciences. Moscow, Science, Vol. 88. P. 126-142 (1976)

8. A. A. Nusinov, V. V. Katyushina, The ability to determine the X-ray class of flares from measurements in the 0.05-0.4 nm band, Heliogeophysical studies, Issue 2, P. 5-8. Electronic resource: http://vestnik.geospace.ru/index.php?id=108 (2012)

9. V. T. Minligareev, Yu. M. Kachanovsky, E. A. Panshin, et al., Creation of a distributed reference base for radiometric equipment of heliogeophysical systems of spacecraft for hydrometeorological purposes, World of Measurements, No. 1. P.51-59 (2016)

10. V. T. Minligareev, Yu. M. Kachanovsky, E. A. Panshin et al. Creation of a reference base for calibrating instruments of the heliogeophysical complex of spacecraft for hydrometeorological purposes, Problems of Electromechanics. Proceedings of VNIIEM, Appendix for Moscow JSC “Corporation VNIIEM,” P.183-192 (2015) 